\title{
Direct Observation of Nucleocytoplasmic Transport by Microinjection of GFP-Tagged Proteins in Living Cells
}

BioTechniques 27:350-355 (August 1999)

\author{
O. Rosorius, P. Heger, \\ G. Stelz, N. Hirschmann, \\ J. Hauber and R.H. Stauber \\ University of Erlangen-Nürn- \\ berg, Erlangen, Germany
}

\begin{abstract}
We established a straightforward experimental system to investigate directly the requirements for nucleocytoplasmic transport in live cells. For this purpose, substrates were created containing nuclear localization signals $(N L S)$ or nuclear export signals (NES) linked to a chimeric protein composed of the glutathione S-transferase (GST) fused to the green fluorescent protein (GFP). The combination of GST/GFP-tagging allowed us to control protein expression in bacteria and to monitor protein purification during chromatography. Following microinjection into somatic cells, nuclear export/import of the highly fluorescent substrates could be observed directly by fluorescence microscopy. This system sets the stage to quantitate, in real time, the kinetics of nuclear import/export in living cells and to evaluate qualitative differences in various NLS/NES signals and pathways.
\end{abstract}

\section{INTRODUCTION}

The controlled transport of molecules and RNAs between the cytoplasm and the nucleus of eukaryotic cells is critical for cellular homeostasis. In general, the nuclear localization of proteins is dependent on their size as well as specific signals mediating nuclear import/export or nuclear/cytoplasmic retention. Proteins with a molecular mass less than $40 \mathrm{kDa}$ are thought to diffuse freely between the nucleus and the cytoplasm in the absence of specific signals. However, even diffusion through the aqueous channel of the nuclear pore complexes (for review see Reference 5) appears to be additionally regulated, e.g., influenced by intracellular calcium concentrations (12).

Nuclear import is mediated by short stretches of basic amino acids, called nuclear localization signals (NLS), which interact with the importin $\alpha / \beta$ axis or directly with alternative import receptors (for detailed reviews see References 4, 6, 8, 9 and 16).

Nuclear export of proteins, on the other hand, is mediated by nuclear export signals (NES), mostly characterized by clusters of leucine residues as identified in a variety of cellular and viral proteins (for reviews see References 4, 6 and 16). An exception is, for example, the M9 domain of the hnRNPA1 protein, a glycine-rich sequence mediating both nuclear import and export (11).

To date, several experimental in vitro and in vivo systems have been established to map NLS/NES sequences and to identify the components required for nucleocytoplasmic trafficking (e.g., see References 1, 3, 10 and 13). However, there is still a need for additional experimental systems to allow study of realtime kinetics of nuclear import/export in living cells. Consequently, we established a straightforward approach to observe directly in living cells the kinetics and requirements of nuclear trafficking.

\section{MATERIALS AND METHODS}

\section{Plasmids}

To generate the bacterial expression vector pGEX-GFP, the coding region of an enhanced green fluorescent protein (GFP) mutant was amplified by polymerase chain reaction (PCR) from the plasmid pCMV-GFPsg25 (14) using appropriate oligonucleotides containing BamHI or EcoRI-restriction sites, respectively. Subsequently, the PCR product was digested with BamHI/EcoRI and cloned into the BamHI/EcoRI-cut bacterial expression vector pGEX-3X (Amersham Pharmacia Biotech, Freiburg, Germany). High-level expression is under the control of the isopropyl- $\beta$ D-thiogalactopyranoside (IPTG) inducible tac promotor/lac operator system. pGEX-GFP codes for a fusion of glutathione S-transferase (GST) linked to GFP (see Figure 1). Plasmid pGEXNES-GFP encodes a GST-nuclear export signal-GFP fusion protein [HTLV-1 Rex amino acids LSAQLYSSLSLD (7)]. To construct this plasmid, comple- 
mentary oligonucleotides were annealed and cloned into the BamHI/NheI-cut vector pGEX-GFP. Likewise, the construct pGEX-NLS-GFP codes for a GST-NLS-GFP fusion protein containing a typical bipartite NLS (amino acids KARRPALRSPPLGTRKRKRPSR) (4) and was constructed by PCR using appropriate oligonucleotides. The PCR product was digested with $\mathrm{BamHI} /$ NheI and cloned into the BamHI/NheI-cut vector $\mathrm{pGEX}$-GFP.

The coding regions of all constructs were confirmed by sequence analysis as previously described (14).

\section{Purification of GST-GFP Fusion Proteins}

GST-GFP hybrid proteins were expressed in Escherichia coli BL21 and affinity-purified from crude lysates with Glutathione-Sepharose ${ }^{\circledR} 4 \mathrm{~B}$ according to the specifications of the manufacturer under non-denaturing conditions (Amersham Pharmacia Biotech). Briefly, $500 \mathrm{~mL}$ exponentially growing cultures were induced with $1 \mathrm{mM}$ IPTG for $4 \mathrm{~h}$ at $33^{\circ} \mathrm{C}$. Bacterial lysates were incubated with $500 \mu \mathrm{L}$ GlutathioneSepharose $4 \mathrm{~B}$ for $1 \mathrm{~h}$ at $4^{\circ} \mathrm{C}$, and the GST-fusion proteins were eluted using $15 \mathrm{mM}$ glutathione. The integrity of the purified proteins was controlled by sodium dodecyl sulfate polyacrylamide gel electrophoresis (SDS-PAGE) and Coomassie ${ }^{\circledR}$ Blue staining. Eluted proteins were dialyzed extensively against cold phosphate-buffered saline (PBS) and stored at $-70^{\circ} \mathrm{C}$.

\section{Microscopy and Microinjection}

Vero cells $\left(2 \times 10^{5}\right)$ were seeded into coated 50-mm glass-bottom dishes (MatTek, Ashland, MA, USA) in Dulbecco's modified Eagle medium (DMEM). One day later, microinjection of the different GST-GFP hybrid proteins (concentration ca. $1.5 \mathrm{mg} / \mathrm{mL}$ ) into the cytoplasm or nucleus was performed using a CompiC INJECT ${ }^{\mathrm{TM}}$ Computer-Assisted Injection System (Cellbiology Trading, Hamburg, Germany). Following injection, cells were immediately observed in PBS with an Axiovert ${ }^{\circledR} 135$ Inverted Fluorescence Microscope (Carl Zeiss, Thornwood, NY, USA). GFP signals were obtained with a fluorescence isothiocyanate (FITC)-fluorescence filter set (Zeiss O9, excitation $450-490 \mathrm{~nm}$, beam splitter $510 \mathrm{~nm}$, emission filter $>520 \mathrm{~nm}$; Carl Zeiss). Images (12-bit) were captured using a cooled MicroMax ${ }^{\mathrm{TM}} \mathrm{CCD}$ camera (Princeton Instruments, Stanford, CA, USA). Image analysis and presentation was performed using the $\mathrm{IPLab}^{\mathrm{TM}}$ software package (Scanalytics, Billerica, MA, USA). The nuclear signal was obtained by measuring the pixel intensity in the nucleus. All pixel values were measured below saturation limits.

\section{RESULTS AND DISCUSSION}

Various systems have been developed to investigate nuclear import/export. Digitonin permeabilized cells serve as an in vitro assay to monitor the nucleocytoplasmic transport of fluorescent-labeled substrates $(1,10)$. In vivo, heterokaryon analysis allows evaluation of the shuttling capacity of proteins, visualized either by indirect immunofluorescence or more elegant by GFP-tagging (2). On the other hand, permeabilized cells are not a biologically intact system, and even heterokaryon analysis is complicated by the simultaneous occurrence of nuclear import and export. Thus, microinjection of transport substrates followed by visualization using indirect immunofluorescence has proven an efficient approach to investigate directly nuclear export independent of import or vice versa (7). However, this method does not allow monitoring the kinetics of import/export directly in living cells. Thus, we combined the advantages of GFP as a live marker with the power of microinjection. In addition, the combination of GST/GFP-tagging enables following and controlling protein expression and purification.

The enhanced GFP mutant used in this study emits green light even without UV-illumination, and thus, the induction time for protein expression, the solubility of the hybrid protein, its binding to the Glutathione-Sepharose and protein elution could be constantly monitored and optimized. The different GST-GFP hybrid proteins were expressed efficiently, were soluble and could easily be purified by chromatography. SDS-PAGE revealed a major single band of approximately $54 \mathrm{kDa}$ (data not shown).

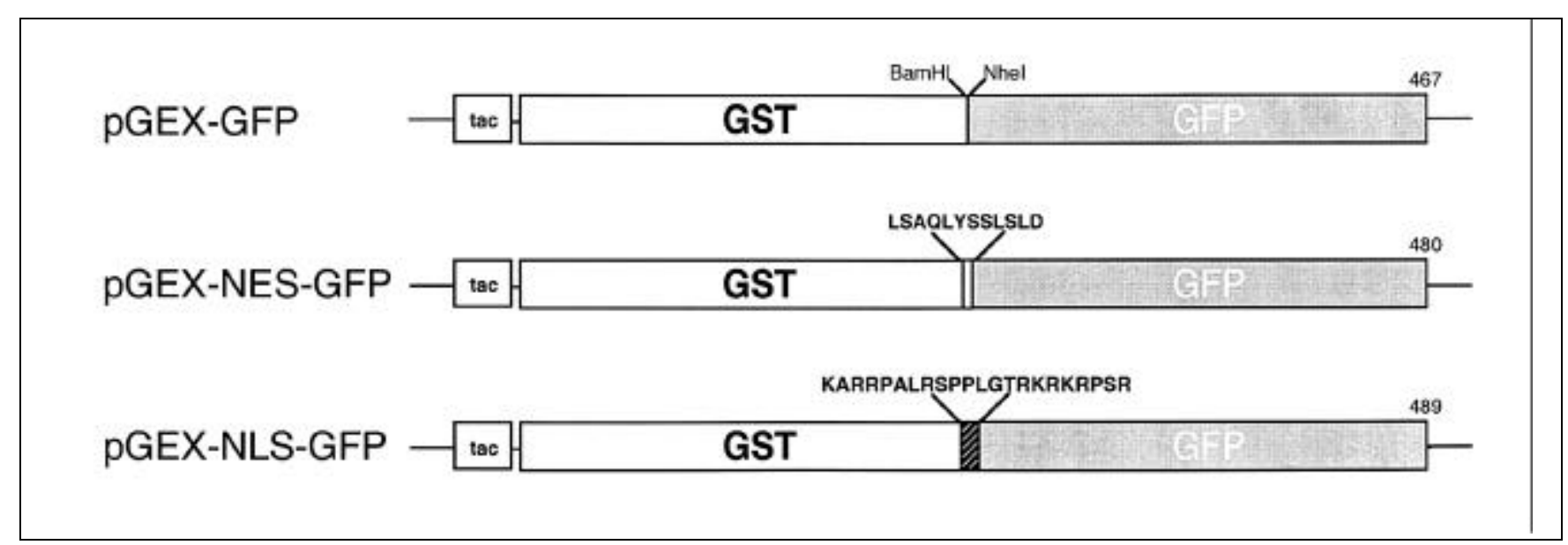

Figure 1. Schematic representation of the different GST-GFP bacterial expression constructs. Expression of the GST-GFP hybrid proteins is under the control of the IPTG-inducible tac promotor/lac operator system. The indicated unique BamHI and NheI restriction sites were used for cloning of the NLS/NES. 


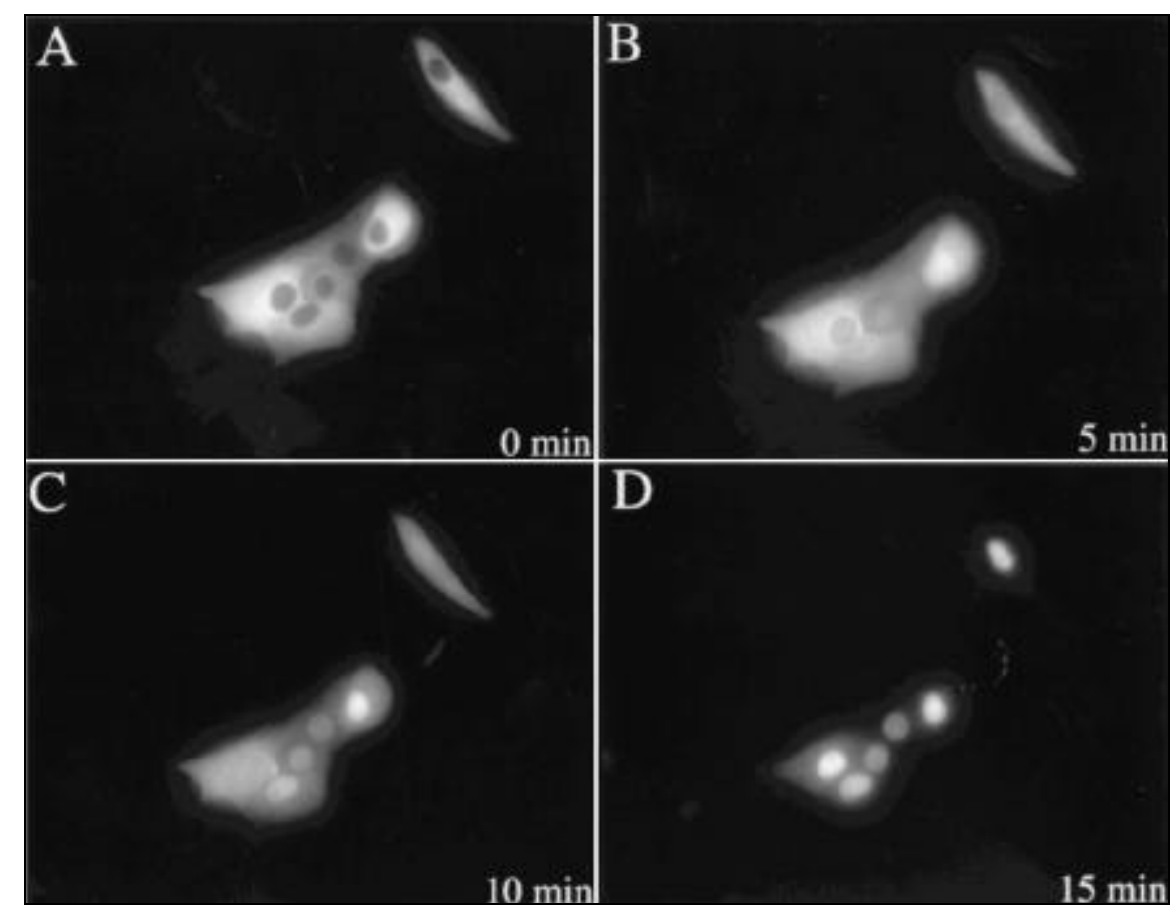

Figure 2. Real-time kinetics of nuclear import of a GST-NLS-GFP hybrid in living cells. Purified GST-NLS-GFP was microinjected into the cytoplasm of Vero cells, and nuclear import was monitored directly by fluorescence microscopy. (A) 0 min post-injection. (B) 5 min post-injection. (C) 10 min postinjection. (D) $15 \mathrm{~min}$ post-injection. Nuclear import occurs rapidly and is completed after $15 \mathrm{~min}$ at $37^{\circ} \mathrm{C}$.

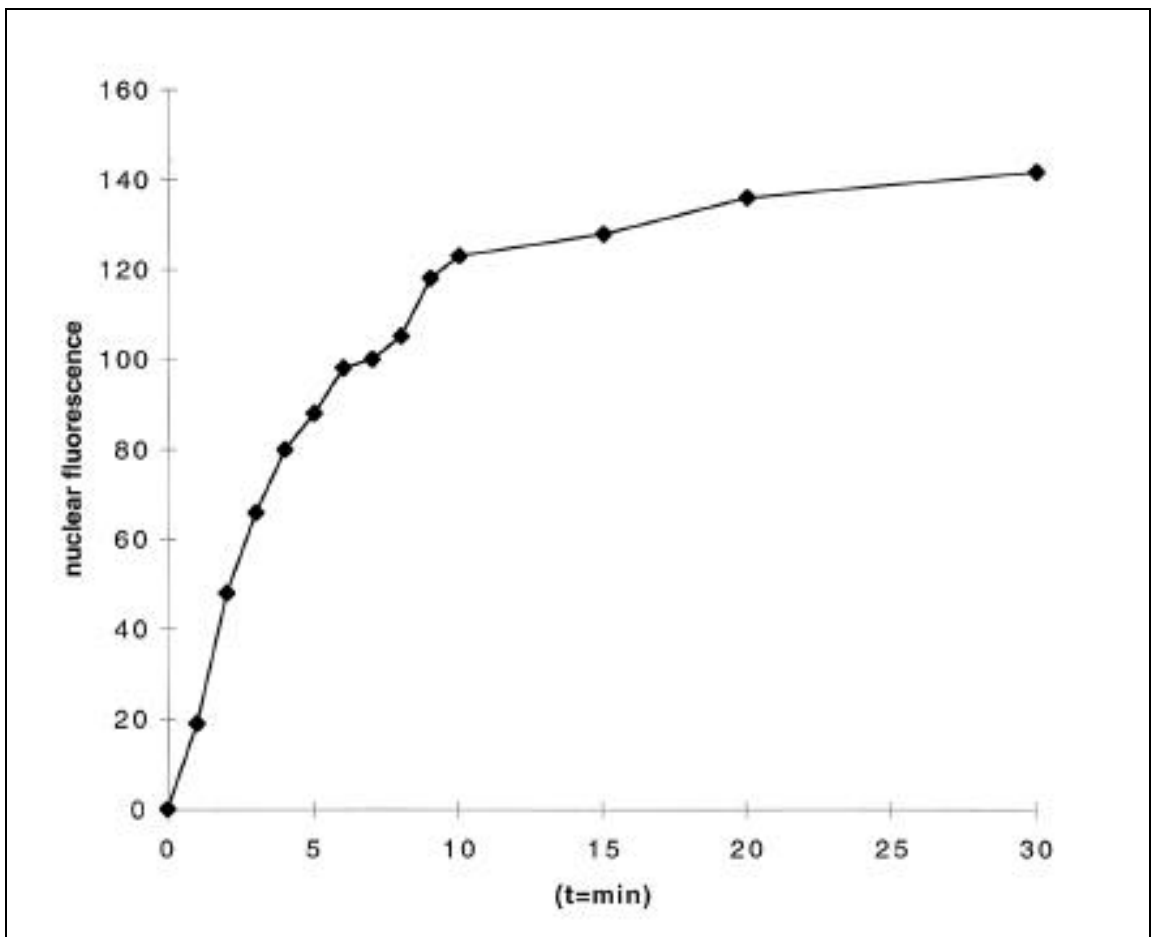

Figure 3. Nuclear import of a GST-NLS-GFP substrate in living cells. Purified GST-NLS-GFP was microinjected into the cytoplasm of Vero cells, and images were recorded at different time points post-injection using a charge-coupled device (CCD) camera. The nuclear signal was obtained by measuring the pixel intensity in the nucleus. All pixel values were measured below saturation limits. Nuclear import occurs rapidly and was saturated after approximately $15 \mathrm{~min}$. 


\section{Research Report}

To investigate if the fluorescent signal of the GFP-hybrid proteins could be detected following microinjection, $5 \mathrm{fL}$ GST-NLS-GFP $(1.5 \mathrm{mg} / \mathrm{mL})$ were injected into the cytoplasm of Vero cells. As shown in Figure 2, the injected substrate could easily be detected in the cytoplasm of live cells, and nuclear import followed over time (Figure 3). After 15 min, import was almost completed in all injected cells. Similar import kinetics were observed in independent experiments (data not shown). As expected, we observed a temperaturedependence of import, since nuclear entry was significantly delayed if the cells were incubated in ice-cold PBS (data not shown). As a control, injection of GST-GFP, lacking a specific import signal, did not result in nuclear localization (see Figure 5, C and D). Likewise, the nuclear export substrate (GST-NES-GFP) was exported efficiently over time following microinjection into nuclei (Figure 4). Again, GSTGFP served as a control and was not exported (see Figure 5, A and B). We observed that GST-GFP remained at the site of injection even after $2 \mathrm{~h}$, indicating that the results obtained in our system are not flawed by passive diffusion (data not shown). In contrast, Chatterjee et al. (3) observed that a hybrid protein composed of two GFPs equilibrated between the nucleus and the cytoplasm following transient expression. Although both substrates (GST-GFP vs. GFP-GFP) are of comparable size (ca. $54 \mathrm{kDa}$ ), the difference between the two systems might be explained by the observation that GST appears to form dimers $(15,17)$. Thus, under the non-denaturing conditions inside the living cell, it is possible that the GST-GFP substrates exist as dimers with a molecular mass of $108 \mathrm{kDa}$ and are therefore less affected by diffusion. Thus, GST-GFP appears to be the preferential system to characterize nuclear import/export signals independent of passive diffusion. Although, we observed some bleaching of GFP after repeated UV-illumination, the signal was stable enough to be detectable even $2 \mathrm{~h}$ post-injection. This degree of signal stability could hardly be achieved using in vitro fluorescent-labeled import substrates, which also appear to be toxic due to the generation of free radicals

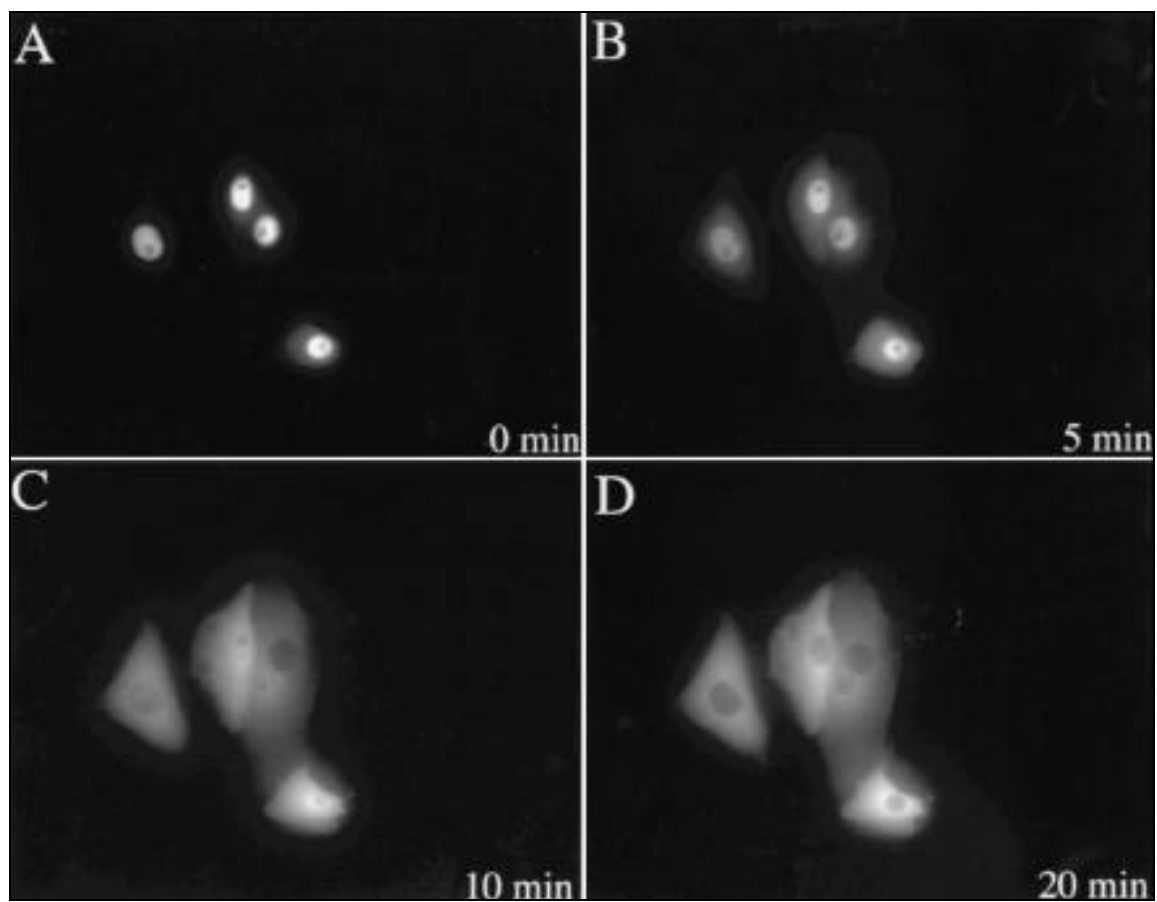

Figure 4. Real-time kinetics of nuclear export of a GST-NES-GFP hybrid in live cells. Purified GSTNES-GFP was microinjected into the nucleus of Vero cells, and nuclear export was monitored directly by fluorescence microscopy in live cells. (A) 0 min post-injection. (B) 5 min post-injection. (C) $10 \mathrm{~min}$ post-injection. (D) 20 min post-injection. Nuclear export occurs and is almost completed after $20 \mathrm{~min}$ at $37^{\circ} \mathrm{C}$
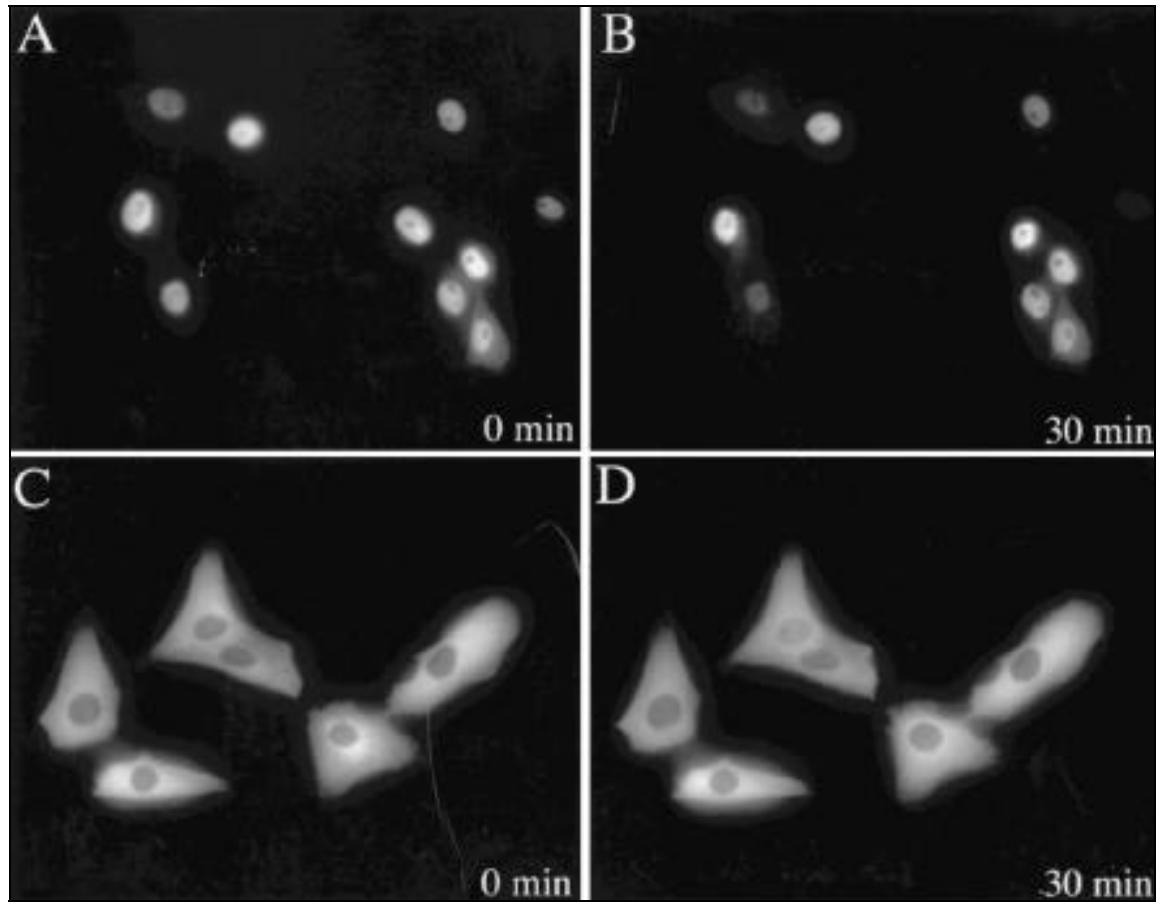

Figure 5. GST-GFP, lacking specific transport signals, is not trafficking. As a control, purified GSTGFP was microinjected into the nucleus (A and B) or cytoplasm (C and D) of Vero cells and monitored by fluorescence microscopy. (A) 0 min post-injection. (B) $30 \mathrm{~min}$ post-injection. (C) 0 min post-injection. (D) 30 min post-injection. GST-GFP remains at the site of injection. 
after prolonged UV-illumination (data not shown). Thus, the GFP-tagged recombinant proteins represent an efficient and cheap alternative to chemically labeled import/export substrates, even for in vitro assays.

In summary, we have established a straightforward approach to express and purify recombinant import/export substrates that can be analyzed directly by microinjection in live cells. This system allows quantification and characterization of real-time kinetics of intracellular transport mediated by various import/export signals to understand and also to interfere with nucleocytoplasmic trafficking.

\section{ACKNOWLEDGMENTS}

O. Rosorius and P. Heger contributed equally to this work. We are indebted to A. Prechtel for excellent data processing and B. Fleckenstein for continuous support. R.S. is supported by the DKFZ AIDS-Stipendium Programm. This work was supported by the Deutsche Forschungsgemeinschaft (Grant No. SFB466).

\section{REFERENCES}

1.Adam, E.J.H. and S.A. Adam. 1994. Identification of cytosolic factors required for nuclear localization and sequence-mediated binding to the nuclear envelope. J. Cell Biol. 125:547-555.

2.Afonina, E., R. Stauber and G.N. Pavlakis. 1998. The human poly-A binding protein 1 shuttles between the nucleus and the cytoplasm. J. Biol. Chem. 273:13015-13021.

3.Chatterjee, S. and U. Stochaj. 1998. Diffusion of proteins across the nuclear envelope of HeLa cells. BioTechniques 24:668-674.

4.Corbett, A. and P.A. Silver. 1997. Nucleocytoplasmic transport of macromolecules. Microbiol. Mol. Biol. Rev. 61:193-211.

5.Doye, V. and E. Hurt. 1997. From nucleoporins to nuclear pore complexes. Curr. Opin. Cell Biol. 9:401-411.

6.Görlich, D. 1998. Transport into and out of the cell nucleus. EMBO J. 17:2721-2727.

7.Heger, P., O. Rosorius, J. Hauber and R.H. Stauber. 1999. Titration of cellular export factors, but not heteromultimerization, is the molecular mechanism of trans-dominant HTLV-1 Rex mutants. Oncogene 18:40804090.

8.Izzaurralde, E. and S. Adam. 1998. Transport of macromolecules between the nucleus and the cytoplasm. RNA 4:351-364.

9.Jäkel, S. and D. Görlich. 1998. Importin $\beta$, transportin, RanBP5 and RanBP7 mediate nu- clear import of ribosomal proteins in mammalian cells. EMBO J. 17:4491-4502.

10.Kehlenbach, R.H., A. Dickmanns and L. Gerace. 1998. Nucleocytoplasmic shuttling factors including Ran and CRM1 mediate nuclear export of NFAT in vitro. J. Cell Biol. 141:863-874.

11.Nakielny, S. and G. Dreyfuss. 1997. Nuclear export of proteins and RNAs. Curr. Opin. Cell Biol. 9:420-429.

12.Perez-Terzic, C., M. Jaconi and D.E. Clapham. 1997. Nuclear calcium and the regulation of the nuclear pore complex. Bioassays 19:787-792.

13.Shulga, N., P. Roberts, Z. Gu, L. Spitz, M. Tabb, M. Nomura and D.S. Goldfarb. 1996. In vivo nuclear transport kinetics in Saccharomyces cerevisiae: a role for heat shock protein 70 during targeting and translocation. J. Cell. Biol. 135:329-339.

14.Stauber, R.H., K. Horie, P. Carney, E.A. Hudson, N.I. Tarasova, G.A. Gaitanaris and G.N. Pavlakis. 1998. Development and applications of enhanced green fluorescent protein mutants. BioTechniques 24:462-471.

15.Tudyka, T. and A. Skerra. 1997. Glutathione S-transferase can be used as a C-terminal, enzymatically active dimerization module for recombinant protease inhibitor, and functionally secreted into the periplasm of Escherichia coli. Protein Sci. 6:2180-2187.

16.Weis, K. 1998. Importins and exportins: how to get in and out of the nucleus. Trends Biochem. Sci. 23:185-189.

17.Yang, L., T. Guan and L. Gerace. 1997. Lamin-binding fragment of LAP2 inhibits increase in nuclear volume during the cell cycle and progression into S phase. J. Cell Biol. 139:1077-1087.

Received 14 December 1998; accepted 29 March 1999.

\section{Address correspondence to:}

Dr. Roland H. Stauber

University of Erlangen-Nuernberg

Institute for Medical and Clinical Virology

Schlossgarten 4

D-91054 Erlangen, Germany

Internet:rdstaube@viro.med.uni-erlangen.de 